\title{
Antenatal and postnatal combined therapy for autoantibody-related congenital atrioventricular block
}

\author{
Antonio Di Mauro ${ }^{1 *}$, Vita Caroli Casavola ${ }^{2}$, Giovanna Favia Guarnieri ${ }^{1}$, Grazia Calderoni ${ }^{1}$, Ettore Cicinelli ${ }^{2}$
} and Nicola Laforgia ${ }^{1}$

\begin{abstract}
Background: Autoantibody-related congenital heart block (CHB) is an autoimmune condition in which trans placental passage of maternal autoantibodies cause damage to the developing heart conduction system of the foetus.

Case presentation: We report a case of an Italian 31-year-old woman, in a good clinical status, referred to our Centre at 26 weeks of her first pregnancy, because of foetal bradycardia, found during routine foetal ultrasonography. Foetal echocardiography revealed a 3rd degree $\mathrm{CHB}$, without any anatomical defects. Despite the mother was asymptomatic for autoimmune disease, anti-Ro/La were searched for, because of the hypothesis of autoantibody-related CHB. High title of maternal anti-Ro/SSA antibodies was found and diagnosis of an autoantibody-related CHB was made. A combination treatment protocol of the mother was started with oral betamethasone, plasmapheresis and IVIG. An emergency C-section was performed at $32+3$ weeks of gestation because of a non-reassuring cardiotocography pattern. A male newborn (BW 1515 g, NGA, Apgar 8-10) was treated since birth with high-flow $\mathrm{O} 2$ for mild RDS. IVIG administration was started at one week, and then every two weeks, until complete disappearance of maternal antibodies from blood. Because of persistent low ventricular rate $(<60 / \mathrm{min})$, seven days following birth, pacemaker implantation was performed. The baby is now at 40th week with no signs of cardiac failure and free of any medications.
\end{abstract}

Conclusion: Up to date, no guidelines have been published for the treatment of "in utero-CHB" and only anecdotal reports are available. It has been stated that a combination therapy protocol is effective in reversing a 2 nd degree CHB, but not for 3rd degree CHB. In cases of foetal bradycardia, weekly foetal echocardiographic monitoring needs to be performed and in cases of 2nd degree CHB and 3rd degree CHB maternal therapy could be suggested, as in our case, to avoid foetal heart failure. In cases of 3rd degree CHB often pacemaker implantation is needed.

Keyword: Autoantibody-associated congenital heart block, Combination therapy protocol of plasmapheresis, intravenous immunoglobulin and betamethasone in congenital heart block, Pacemaker implantation in newborn, Foetal cardiac arrhythmia detection, Foetal ecochardiography, Neonatal counselling

\footnotetext{
*Correspondence: dimauroantonio@msn.com

'Department of Biomedical Science and Human Oncology, Neonatology and Neonatal Intensive Care Unit, University of Bari, "Aldo Moro", P.zza Giulio

Cesare, 11, 70125 Bari, Italy

Full list of author information is available at the end of the article
} 


\section{Background}

Autoantibody-associated congenital heart block (CHB) is a rare neonatal disease with an overall prevalence of $1 / 20000$ liveborn [1]. It may be detected in utero as a 1st- or 2nd-degree atrioventicular (AV) block, but most of the affected foetuses have a potentially lethal 3rddegree, complete AV block [2]. Occasionally is associated a life-threatening cardiomyopathy [3].

Reported perinatal mortality rate is about $20-30 \%$ and approximately $57-66 \%$ of children born alive with CHB require pacemaker before reaching adulthood [4].

Autoantibody-associated CHB is considered a model of passively acquired autoimmune disease in which the trans-placental passage of maternal antinuclear antibodies (ANA) causes immune-mediated inflammation of the developing myocardial tissue and conduction system of the foetus [5].

Approximately $85 \%$ of foetus with congenital heart block and absence of structural abnormalities have maternal transfer of antibodies against SSA/Ro and SSB/La [6]; however only $2 \%$ of seropositive mother have newborns with congenital heart block [7]. This low risk rate rises to $19 \%$ for women with a previously affected newborn [8]. According to these data, antibodies to SSA/Ro and SSB/La could not be the only cause of the disease and other maternal and foetal factors are important [9]. Nevertheless, maternal health status is not considered a risk factor for CHB; approximately 40-60\% of mothers with an affected newborn are totally asymptomatic for autoimmune disease when foetal bradycardia is found [10].

Clinical signs of conduction abnormalities (1st, 2nd, 3rd-degree heart block) most commonly develop during 18-24 weeks of pregnancy and may be found by foetal Doppler echocardiography [11]. CHB is considered a progressively developing disease and 3rd-degree heart block appears to be irreversible. Nevertheless, anecdotal cases of antenatal therapy describe the possibility of complete regression of 1st and 2nd -degree heart blocks, but only a stop of progression to heart failure for 3rd-degree heart blocks $[12,13]$.

Up to date, no therapy has demonstrated in large case studies to be effective in preventing the progression of heart injury and in reversing autoantibody-associated CHB. We report the outcome of a combination therapy protocol described in detail in a recent paper by Ruffatti et al. [12] to treat a case of autoantibody-related 3rddegree heart blocks referred to our Neonatal Intensive Care Unit.

\section{Case presentation}

A healthy, primigravida, asymptomatic 31-year-old woman was referred to our Obstetric Unit at 26 weeks of gestation, because of the finding of foetal bradycardia during routine obstetric ultrasonography examination. The foetal echocardiography, performed in our clinic, revealed dissociation between atrial rhythm $(154 / \mathrm{bpm})$ and ventricular rhythm (54 bpm) (Figure 1). Neither structural heart defects nor hydrops fetalis were found.

Despite the mother was asymptomatic for any autoimmune diseases, anti-Ro/La autoantibodies were searched for, because of the possibility of autoantibodies-related CHB. High title of maternal anti-Ro/SSA was found (359,5 $\mathrm{U} / \mathrm{ml}$ ) and diagnosis of an autoantibody-related $\mathrm{CHB}$ was made. After prenatal counselling between neonatologists, cardiologists, rheumatologists and obstetricians, mother started a combination therapy protocol of plasmapheresis, intravenous immunoglobulin and betamethasone (Figure 2). Foetal heart rate remained stable until the $32+3$ weeks of gestation, when a non-reassuring cardiotocography pattern occurred and a $1515 \mathrm{~g}$ male was delivered by an emergency C- section. A male newborn (1515 g, NGA, Apgar 8-10) was treated since birth with high-flow O2 for mild RDS. Electrocardiography confirmed complete AV block, with an atrial rate of $136 \mathrm{bpm}$ and a ventricular rate of $44 \mathrm{bpm}$ (Figure 3), and no significant heart rate increase after isoproterenol (up to $0,1 \mu \mathrm{g} / \mathrm{kg} / \mathrm{min}$ ) administration. Newborn serum was positive for ENA $(\mathrm{SSA} / \mathrm{Ro}=257 \mathrm{U} / \mathrm{ml})$ and IVIG administration was started at one week and every two weeks, until complete disappearance of maternal antibodies from blood. Because of persistent low ventricular rate $(<60 / \mathrm{min})$, seven days following birth, pacemaker implantation was performed (Figures 3 ). Because of gestational age $(33 w+3)$ and low weight $(1380 \mathrm{~g})$, the pacemaker (MICRONY TM II SR+; St.Jude Medical) was placed in an abdominal pocket, through a left xipho-umbilican paramedian incision, between the rectus muscle sheet and the posterior surface of the abdominal muscle, as already reported [14,15]. A $35 \mathrm{~cm}$ bipolar steroid-eluting epicardial pacing lead was positioned on the right ventricular free wall through a left

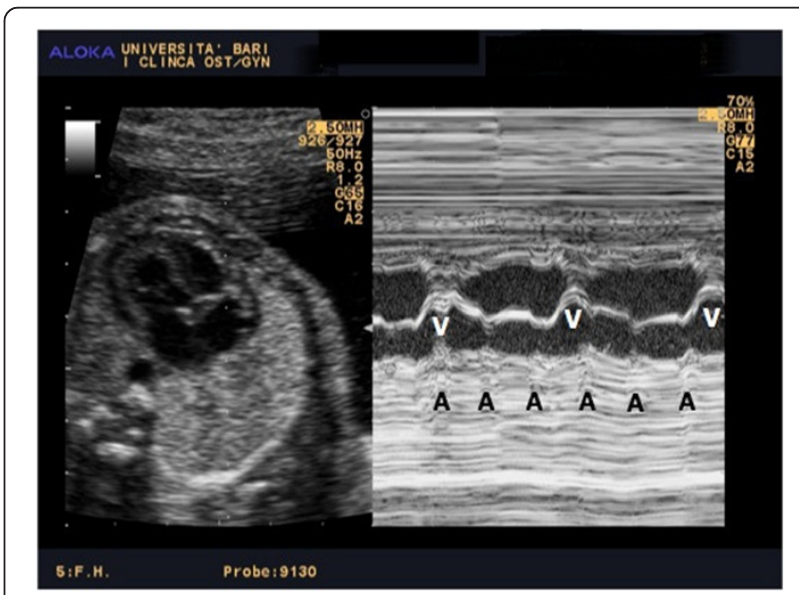

Figure 1 Ultrasonograms of two-dimensional foetal echocardiograpy. Atrial (A) and ventricular (V) contractions. 


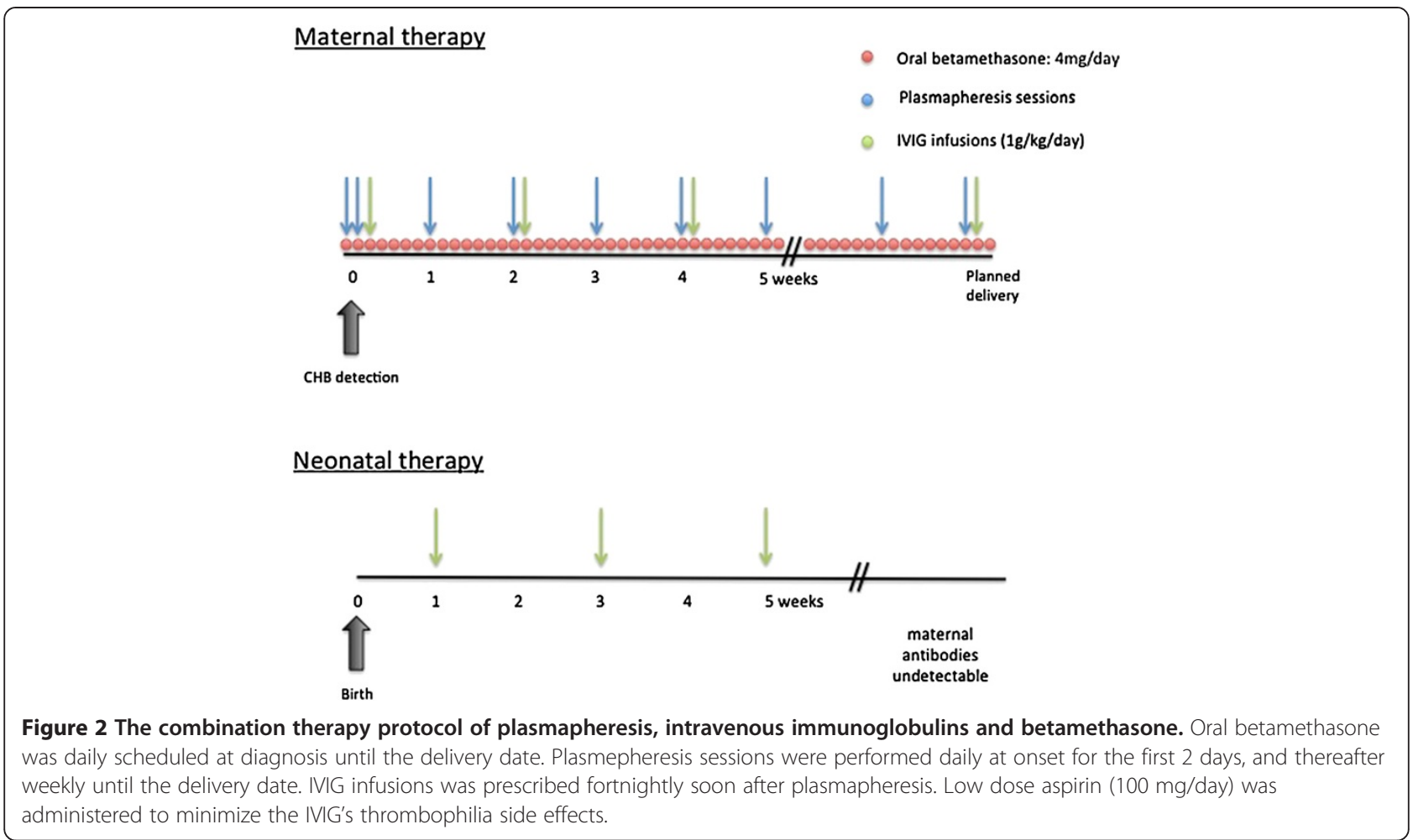

antero-lateral thoracothomy at the 5th intercostal space. The exuberant length of the cable was rolled into the left pleural space, tunneled till the abdominal pocket and connected to the generator. The pacemaker was programmed with a heart rate of $120 \mathrm{bpm}$. The baby is now 40th week with no signs of cardiac failure and free of any medications.

\section{Discussion}

Autoantibody-related $\mathrm{CHB}$ is an autoimmune condition in which trans-placental passage of maternal autoantibodies cause damage to the developing heart conduction system of the foetus. Up to date, no therapy is found to be effective in the treatment of 2nd and 3rd degree $\mathrm{CHB}$. Ruffatti et al. have recently showed the possibility

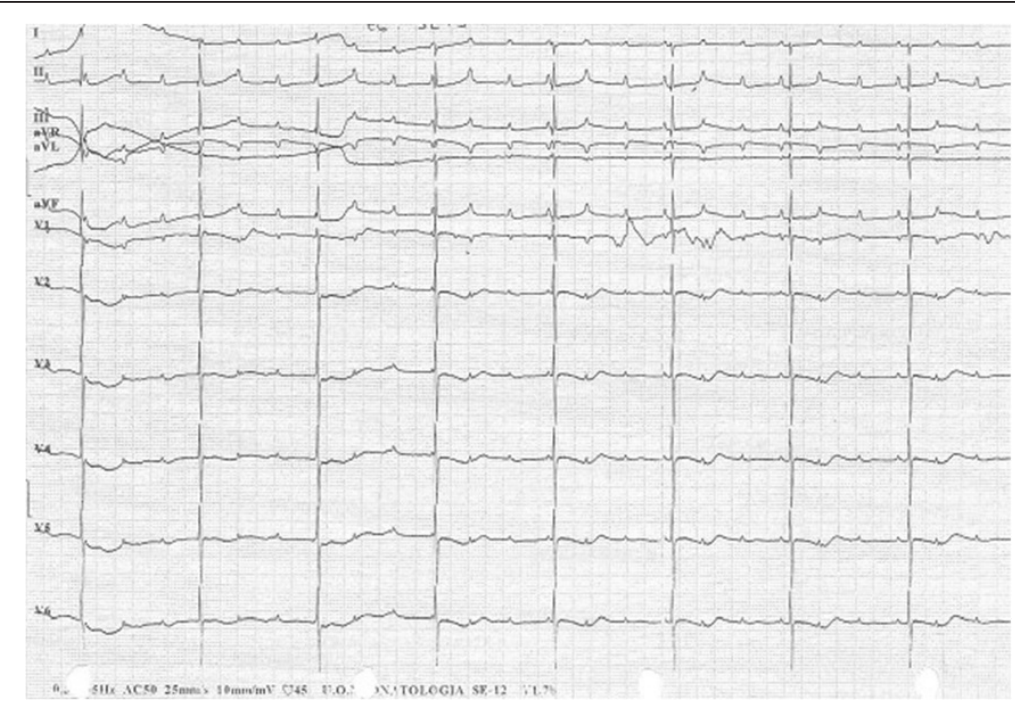

Figure 3 A neonatal electrocardiography shows complete atrioventricular block, with an atrial rate of $136 \mathrm{bpm}$ and a ventricular rate of $44 \mathrm{bpm}$. 
of a combination strategy in order to revert or block the progression of immune-related heart damage [12,13]. The rationale behind this combined protocol is that it is possible to obtain a sum of the effects of each drug: a) bethametasone, a fluorinated steroids only partially inactivated by the placenta, reduces inflammation of the developing conduction system; b) plasmapheresis lowers the level of the offending antibodies; c) IVIG made an anti-idiotype regulation, inhibiting the placental transport of maternal antibodies, and modulating macrophages response with secondary reduction of inflammation and fibrosis of the foetal heart.

This is a report of an antibody-related complete CHB, treated in utero with a combination therapy protocol, a pacemaker implantation at one week of age and bi-weekly IVIG administration to the newborn.

According to the protocol, the newborn was also treated with IVIG after birth, and every two weeks, until maternal autoantibodies became undetectable. The treatment continued in post-natal period because, although the pacemaker implantation preserve electrophysiological heart failure, it does not avoid myocardial injury from persistent maternal autoantibodies that may clinically manifests with myocardial dysfunction after birth, even with adequate pacemaker therapy [16]. We have also avoided breastfeeding, because of the possible transfer human of anti-Ro/La antibodies via breast milk and subsequent tissue injury [17]. This post-natal treatment may accelerate the clearance of potentially pathogenic autoantibodies and avoid the risk of myocardial injury (fibrosis) progression.

No side effects related to the protocol were observed both in the mother and newborn. Despite the case reported by Ruffatti et al. [12] showed an improvement of foetal heart rate, we were not able to demonstrate this effect in our foetus with 3rd degree AV block, and an early pacemaker implantation was needed after birth, at one week of age. Probably, the timing of starting the combined protocol during pregnancy might affect the result in term of foetal heart rate improvement. Further studies are needed to demonstrate the correct timing and efficacy of this combined protocol.

\section{Conclusion}

Different therapeutic regimens have been proposed for foetal/neonatal $\mathrm{CHB}$, but controlled clinical trials are not yet available. CHB is a progressive disease and, presumably, the best time for any therapy is during early stages of pregnancy, when inflammation of the heart conduction system, but not fibrosis, is present. Thus, early assessment of the foetus and serial foetal echocardiographs to identify reversible block are needed, to start treatment when $\mathrm{CHB}$ is still incomplete [18]. Multidisciplinary prenatal counseling is fundamental for the best treatment, both for foetus and newborn.

\section{Consent}

Written informed consent was obtained from the patient for publication of this case report and any accompanying images. A copy of the written consent is available for review by the Editor of this journal.

\section{Abbreviations \\ CHB: Congenital heart block; AV: Atrioventricular; Bpm: Beat per minute: ANA: Antinuclear antibodies.}

\section{Competing interest}

The authors declare that they have no competing interest.

\section{Authors' contributions}

GC and VCC participated in the literature search and in selected relevant papers. GFG resolved inconsistencies with discussion. NL and EC was involved in revising the final draft of the manuscript. ADM was responsible for the concept, design and writing of the final version of the manuscript. All authors read and approved the final manuscript.

\section{Acknowledgements}

The authors would like to thank the whole ONSP team for having awarded this clinical case during the National Congress ONSP DAYS 2013 (Cagliari, 5-8 June). ONSP is the Italian national council of pediatric residents and fellows.

\section{Author details}

'Department of Biomedical Science and Human Oncology, Neonatology and Neonatal Intensive Care Unit, University of Bari, "Aldo Moro", P.zza Giulio Cesare, 11, 70125 Bari, Italy. ${ }^{2}$ Department of Gynecology and Obstetrics, University of Bari "Aldo Moro", P.zza Giulio Cesare, 11, 70125 Bari, Italy.

Received: 4 June 2013 Accepted: 23 November 2013 Published: 29 November 2013

\section{References}

1. Buyon JP, Clancy RM: Neonatal lupus: basic research and clinical perspectives. Rheum Dis Clin North Am 2005, 31:299-313.

2. Strasburger JF, Wakai RT: Fetal cardiac arrhythmia detection and in utero therapy. Nat Rev Cardiol 2010, 7(5):277-290.

3. Nield LE, Silverman ED, Taylor GP, Smallhorn JF, Mullen JB, Silverman NH, Finley JP, Law YM, Human DG, Seaward PG, Hamilton RM, Hornberger LK: Maternal anti-Ro and anti-La antibody-associated endocardial fibroelastosis. Circulation 2002, 105(7):843-848.

4. Hon KL, Leung AK: Neonatal lupus erythematosus. Autoimmune Dis. 2012, 2012:301274

5. Carvalheiras G, Faria R, Braga J, Vasconcelos C: Fetal outcome in autoimmune diseases. Autoimmun Rev 2012, 11(6-7):A520-A530.

6. Jaeggi ET, Hornberger LK, Smallhorn JF, Fouron JC: Prenatal diagnosis of complete atrioventricular block associated with structural heart disease: combined experience of two tertiary care centers and review of the literature. Ultrasound Obstet Gynecol 2005, 26:16-21.

7. Buyon JP, Clancy RM, Friedman DM: Autoimmune associated congenital heart block: integration of clinical and research clues in management of the maternal/fetal dyad at risk. J Intern Med 2009, 265:653-662

8. Friedman DM, Kim MY, Copel JA, Davis C, Phoon CK, Glickstein JS, Buyon JP: Utility of cardiac monitoring in fetuses at risk for congenital heart block: the PR interval and dexamethasone evaluation prospective study. Circulation 2008, 117:485-493.

9. Ambrosi A, Wahren-Herlenius M: Congenital heart block: evidence for a pathogenic role of maternal autoantibodies. Arthritis Research and Therapy 2012, 14:208

10. Rivera TL, Izmirly PM, Birnbaum BK, Byrne P, Brauth JB, Katholi M, Kim MY, Fischer J, Clancy RM, Buyon JP: Disease progression in mothers of children enrolled in the Research Registry for Neonatal Lupus. Ann Rheum Dis 2009, 68(6):828-835

11. Tomek V, Janoušek J, Reich O, Gilík J, Gebauer RA, Skovránek J: Atrioventricular conduction time in fetuses assessed by Doppler echocardiography. Physiol Res 2011, 60(4):611-616.

12. Ruffatti A, Marson P, Svaluto-Moreolo G, Marozio L, Tibaldi M, Favaro M, Calligaro A, Grava C, Hoxha A, Pengo V, Punzi L: A combination therapy 
protocol of plasmapheresis, intravenous immunoglobulins and betamethasone to treat anti-Ro/La-related congenital atrioventricular block. A case series and review of literature. Autoimmun Rev 2013, 12(7):768-773.

13. Ruffatti A, Milanesi O, Chiandetti L, Cerutti A, Gervasi MT, De Silvestro G,

Pengo V, Punzi L: A combination therapy to treat second-degree anti-Ro/ La-related congenital heart block: a strategy to avoid stable third-degree heart block? Lupus 2012, 21(6):666-671.

14. Roubertie F, Le Bret E, Thambo JB, Roques X: Intra-diaphragmatic pacemaker implantation in very low weight premature neonate. Interact Cardiovascular Thorac Surgery 2009, 9:743-745,

15. Di Mauro A, Ciccone MM, Schettini F, Frasso G, Zito A, Troise D, Annecchino P, Di Mauro F, Scicchitano P, Laforgia N: Permanent pacemaker implantation in $1200 \mathrm{~g}$-preterm suffering from congenital atrioventricular block. EMBJ 2013, 8(19):119.121.

16. Capone C, Buyon JP, Friedman DM, Frishman W: Cardiac manifestations of neonatal lupus: a review of autoantibody associated congenital heart block and its impact in an adult population. Cardiol Rev 2012, 20(2):72-76.

17. Askanase AD, Miranda-Carus ME, Tang X, Katholi M, Buyon JP: The presence of $\mathrm{IgG}$ antibodies reactive with components of the SSA/Ro-SSB/La complex in human breast milk: implications in neonatal lupus. Arthritis Rheum 2002, 46(1):269-271.

18. Bergman G, Wahren-Herlenius M, Sonesson SE: Diagnostic precision of Doppler flow echocardiography in fetuses at risk for atrioventricular block. Ultrasound Obstet Gynecol 2010 Nov, 36(5):561-566.

doi:10.1186/1471-2393-13-220

Cite this article as: Di Mauro et al:: Antenatal and postnatal combined therapy for autoantibody-related congenital atrioventricular block. BMC Pregnancy and Childbirth 2013 13:220.

\section{Submit your next manuscript to BioMed Central and take full advantage of:}

- Convenient online submission

- Thorough peer review

- No space constraints or color figure charges

- Immediate publication on acceptance

- Inclusion in PubMed, CAS, Scopus and Google Scholar

- Research which is freely available for redistribution 